\title{
THE LOWER VARIANCE BOUND OF THE DISORDER MOMENT ESTIMATOR FOR THE NEgative BINOMIAL AND POISSON PROCESSES
}

We discuss the negative binomial process which changes its intensity stepwise at a certain moment of time (the moment of disorder). The Cramer-Rao inequality is usually used to determine the accuracy of parameter estimation. The application of this inequality requires, however, the density function to fulfil particular conditions of regularity. The necessary conditions, which must be satisfied by the density of distribution, are not fulfilled in the problem in which the moment of a stepwise change of the statistical characteristic of a signal is the estimated parameter.

Basing on the inequality proposed by Kiefer, we find the accurate lower variance bound of the disorder moment estimator. In our calculations we use the method presented in [2].

1. We consider the negative binomial process in a given period $[0, T]$. It is a homogeneous process with independent increments for which

$$
P\left(X_{t}(\omega)=k\right)=\frac{\Gamma(t+k)}{\Gamma(t) \Gamma(k+1)} \frac{\lambda^{k}}{(1+\lambda)^{t+k}},
$$

Where $\lambda>0, k=0,1,2, \ldots$ We assume that the process is separable and that almost all sample functions are right continuous. Almost all sample functions of this process are nondecreasing and integral-valued but the magnitudes of jumps may be different. The process changes its intensity $\lambda$ at the moment of time $\vartheta$ (the moment of disorder):

$$
\lambda(t / \vartheta)=a+b \cdot \boldsymbol{1}(t-\vartheta), \quad t \in[0, T], \vartheta \in[0, T], a>0, a+b>0,
$$

where

$$
\boldsymbol{I}(t)= \begin{cases}1 & \text { for } t \geqslant 0 \\ 0 & \text { for } t<0\end{cases}
$$

3-Zastos. Mat. 18.3 
From the properties of the process it follows that every sample function is determined by the sequence of time coordinates $t_{1}, t_{2}, \ldots, t_{n}$ in which jumps bave occurred and by the sequence $k_{1}, k_{2}, \ldots, k_{n}$ of magnitudes of jumps related to this sequence.

We introduce the system of densities $p_{n}\left(t_{1}, k_{1}, \ldots, t_{n}, k_{n}, \vartheta\right)$, which characterizes the process by the probabilities, to obtain jumps that have successively values $k_{1}, \ldots, k_{n}$ of units only in the intervals $\left[t_{1}, t_{1}+\Delta t_{1}\right), \ldots$ $\ldots,\left[t_{n}, t_{n}+\Delta t_{n}\right)$, respectively, with the fixed parameter $\vartheta \in[0, T]$. We denote these probabilities as follows:

$$
P\left(t_{1}, \Delta t_{1}, k_{1}, \ldots, t_{n}, \Delta t_{n}, k_{n}, \vartheta\right)
$$

where $0<t_{i} \leqslant T$ for $i=1, \ldots, n, n=1,2, \ldots, t_{i} \neq t_{j}$ if $i \neq j, \Delta t_{i}>0$ for $i=1, \ldots, n$, and $k_{i} \geqslant 1$ for $i=1, \ldots, n$.

Using the definition of the process and the intensity function we can easily calculate that

$$
\begin{aligned}
& P\left(t_{1}, \Delta t_{1}, k_{1}, \ldots, t_{n}, \Delta t_{n}, k_{n}, \vartheta\right) \\
= & \left(\frac{1}{1+a}\right)^{\vartheta}\left(\frac{1}{1+a+b}\right)^{T-\vartheta} \prod_{i=1}^{n} \frac{1}{k_{i}}\left(\frac{\lambda\left(t_{i} / \vartheta\right)}{1+\lambda\left(t_{i} / \vartheta\right)}\right)^{k_{i}} \Delta t_{i}+o\left(\Delta t_{1} \ldots \Delta t_{n}\right)
\end{aligned}
$$

Hence we obtain the system of densities

$$
\begin{aligned}
p_{n}\left(t_{1}, k_{1}, \ldots, t_{n}, k_{n}, \vartheta\right) \\
=\left(\frac{1}{1+a}\right)^{\vartheta}\left(\frac{1}{1+a+b}\right)^{T-\vartheta} \prod_{i=1}^{n} \frac{1}{k_{i}}\left(\frac{\lambda\left(t_{i} / \vartheta\right)}{1+\lambda\left(t_{i} / \vartheta\right)}\right)^{k_{i}}
\end{aligned}
$$

where $0<t_{i} \leqslant T$ for $i=1, \ldots, n, n=1,2, \ldots, t_{i} \neq t_{j}$ if $i \neq j$, and $k_{i} \geqslant 1$ for $i=1, \ldots, n$.

We find a method to sum up the events whose probability is given by (1.2). The following identity is useful for this purpose:

$$
\sum_{k=n}^{\infty} \sum_{\substack{k_{1}+\ldots, k_{n=k}=1 \\ k_{1} \geqslant 1, \ldots, k_{n} \geqslant 1}} \prod_{i=1}^{n} \frac{1}{k_{i}}\left(\frac{\lambda_{i}}{1+\lambda_{i}}\right)^{k_{i}}=\prod_{i=1}^{n} \ln \left(1+\lambda_{i}\right)
$$

where for each $n(n \geqslant 1)$ the system of numbers $\lambda_{i}(i=1, \ldots, n)$ fulfils the inequality $\lambda_{i} \geqslant-\frac{1}{2}$.

The proof of (1.4), which can easily be carried out by induction, is omitted. Using now identity (1.4) we see that the following equality is 
true:

$$
\begin{aligned}
&\left(\frac{1}{1+a}\right)^{\vartheta}\left(\frac{1}{1+a+b}\right)^{T-\vartheta}+ \\
&+\sum_{n=1}^{\infty} \frac{1}{n !} \int_{0}^{T}(n) \int_{0}^{T}\left\{\sum_{\substack{k=n \\
k_{1}+\ldots+k_{n}=k \\
k_{1} \geqslant 1, \ldots, k_{n} \geqslant 1}}^{\infty}\left(\frac{1}{1+a}\right)^{\vartheta}\left(\frac{1}{1+a+b}\right)^{T-\vartheta} \times\right. \\
&\left.\quad \times \prod_{i=1}^{n} \frac{1}{k_{i}}\left(\frac{\lambda\left(t_{i} / \vartheta\right)}{1+\lambda\left(t_{i} / \vartheta\right)}\right)^{k_{i}}\right\} d t_{1} \ldots d t_{n}=1 .
\end{aligned}
$$

2. The probability density functions (1.3) cannot be everywhere differentiated with respect to the parameter $\vartheta$. We introduce an inequality of the Cramer-Rao type, given in [1], which does not require the conditions of regularity to be satisfied.

Let $X$ be a random variable with density $f(x, \vartheta)(x \in \mathscr{X}, \vartheta \in \Theta)$ with respect to some fixed $\sigma$-finite measure $\lambda$ and let $\Theta \subset \boldsymbol{R}$. For each $\vartheta \in \Theta$, we put

$$
\Theta_{\vartheta}=\{h: \vartheta+h \in \Theta\}
$$

For fixed $\vartheta$, let $\mu_{1}$ and $\mu_{2}$ be two probability densities on $\Theta_{\vartheta}$ such that the integrals

$$
\int_{\Theta_{\vartheta}} h_{h} \mu_{i}(h) d h
$$

exist and are finite for $i=1,2$. Then for any unbiased estimator $t(x)$ of the parameter $\vartheta \in \Theta$ the following inequality holds:

$$
\mathrm{E}_{\vartheta}(t-\vartheta)^{2} \geqslant \sup \frac{\left(\int_{\vartheta} h \mu_{1}(h) d h-\int_{\Theta_{\vartheta}} h \mu_{2}(h) d h\right)^{2}}{\int_{\mathscr{X}} f^{-1}(x, \vartheta)\left(\int_{\vartheta} f(x, \vartheta+h)\left[\mu_{1}(h)-\mu_{2}(h)\right] d h\right)^{2} d \lambda(x)}
$$

where for each $\vartheta$ the supremum is taken over all $\mu_{1}$ and $\mu_{2}$ for which $\mu_{1} \neq \mu_{2}$ and for which the integrand of the integral over $\mathscr{X}$ is defined $\lambda$-a.e.

Inequality (2.1) holds without any restriction on the probability density $f(x, \vartheta)$ and the right-hand side of this inequality is the best possible lower variance bound of the estimator of the parameter $\vartheta$ (see [1]). The right-hand side of inequality (2.1) can be calculated by the method proposed in [2].

We introduce the following symbols:

$$
\begin{gathered}
\mu(h) \stackrel{\text { df }}{=} \mu_{1}(h)-\mu_{2}(h), \\
\Phi_{1}[\mu] \stackrel{\text { df }}{=} \int_{\Theta_{\vartheta}} h \mu(h) d h, \quad \Phi_{2}[\mu] \stackrel{\text { df }}{=} \int_{\mathscr{X}} \frac{1}{f(x, \vartheta)}\left(\int_{\Theta_{\vartheta}} f(x, \vartheta+h) \mu(h) d h\right)^{2} d \lambda(x) .
\end{gathered}
$$


In order to find the supremum in (2.1) we have to find $\mu$ such that

with the restrictions

$$
\sup _{\mu^{\prime}} \frac{\left(\Phi_{1}\left[\mu^{\prime}\right]\right)^{2}}{\Phi_{2}\left[\mu^{\prime}\right]}=\frac{\left(\Phi_{1}[\mu]\right)^{2}}{\Phi_{2}[\mu]}
$$

$$
\begin{gathered}
\int_{\Theta_{\vartheta}} \mu(h) d h=0, \\
\mu(h) \neq 0, \quad h \in \Theta_{\vartheta} .
\end{gathered}
$$

The restriction (2.3) follows from the fact that $\mu_{1}$ and $\mu_{2}$ are the probability densities.

Changing the order of integration in the functional $\Phi_{2}$ we get

$$
\Phi_{2}[\mu]=\int_{\Theta_{\vartheta}} \int_{\Theta_{\vartheta}} K\left(h, h^{\prime}\right) \mu(h) \mu\left(h^{\prime}\right) d h d h^{\prime},
$$

where the kernel $K(\cdot, \cdot)$ is of the form

$$
K\left(h, h^{\prime}\right) \stackrel{d f}{=} \int_{\mathscr{x}} \frac{f(x, \vartheta+h) f\left(x, \vartheta+h^{\prime}\right)}{f(x, \vartheta)} d \lambda(x), \quad h, h^{\prime} \in \Theta_{\vartheta} .
$$

According to [2] the problem defined in (2.2)-(2.3') leads to the Euler equation

$$
\int_{\theta_{\vartheta}} K\left(h, h^{\prime}\right) \mu\left(h^{\prime}\right) d h^{\prime}=\lambda_{1} h+\lambda_{2},
$$

where $\lambda_{1}$ and $\lambda_{2}$ are Lagrange multipliers. From (2.3) it follows that $\lambda_{2}=0$. The multiplier $\lambda_{1}$ can be equal to one, which results from the fact that if the function $\mu(\cdot)$ fulfils condition (2.2), so does the function $c \mu(\cdot)$.

3. Our considerations from Section 2 will be used for obtaining the probability density functions. The kernel (2.5) can be transformed in the following way:

$$
\begin{aligned}
K\left(h, h^{\prime}\right)= & (1+a)^{-\vartheta-h^{\prime}-h}(1+a+b)^{h+h^{\prime}+\vartheta-T} \times \\
& \times\left\{\sum_{n=1}^{\infty} \frac{1}{n !} \sum_{\substack{k=n \\
k_{1}+\ldots+k_{n}=k \\
k_{1} \geqslant 1, \ldots, k_{n} \geqslant 1}}^{\infty} \prod_{i=1}^{n} \frac{1}{k_{i}} I_{k_{i}}\left(\vartheta, h, h^{\prime}\right)+1\right\}
\end{aligned}
$$

for $h, h^{\prime} \in \Theta_{\vartheta}=[-\vartheta, T-\vartheta]$, where

$$
I_{k}\left(\vartheta, h, h^{\prime}\right) \stackrel{\mathrm{df}}{=} \int_{0}^{T} \frac{\left(\frac{\lambda(t / \vartheta+h)}{1+\lambda(t / \vartheta+h)}\right)^{k}\left(\frac{\lambda\left(t / \vartheta+h^{\prime}\right)}{1+\lambda\left(t / \vartheta+h^{\prime}\right)}\right)^{k}}{\left(\frac{\lambda(t / \vartheta)}{1+\lambda(t / \vartheta)}\right)^{k}} d t
$$

for $\vartheta \in[0, T], h, h^{\prime} \in \Theta_{\vartheta}$, and $k=1,2, \ldots$ To find the kernel we uso identity (1.4). 
After integrating and summing (3.1) we obtain the following values of the kernel in the domain $h^{\prime} \geqslant h$ :

$$
K\left(h, h^{\prime}\right)= \begin{cases}\left(\frac{(a+b)(1+a+b)}{a^{2}+a+b+2 a b}\right)^{h} & \text { for } h>0 \\ 1 & \text { for } h \leqslant 0, h^{\prime} \geqslant 0 \\ \left(\frac{a^{2}+a-b^{2}}{(1+a) a}\right)^{h^{\prime}} & \text { for } h^{\prime}<0, h<0 .\end{cases}
$$

This formula holds under the conditions

$$
a^{2}+a-b^{2}>0 \text { and } a^{2}+a+b+2 a b>0
$$

which are necessary for identity (1.4) to hold.

In the remaining domain of changes of $a$ and $b$ we obtain the following values of the kernel for $h^{\prime} \geqslant h$ :

$$
K\left(h, h^{\prime}\right)= \begin{cases}\left(\frac{(a+b)(1+a+b)}{a^{2}+a+b+2 a b}\right)^{h} & \text { for } h>0 \\ 1 & \text { for } h \leqslant 0, h^{\prime} \geqslant 0 \\ \infty & \text { for } h^{\prime}<0\end{cases}
$$

if $a^{2}+a-b^{2} \leqslant 0$, and

$$
K\left(h, h^{\prime}\right)= \begin{cases}\infty & \text { for } h>0 \\ 1 & \text { for } h \leqslant 0, h^{\prime} \geqslant 0 \\ \left(\frac{a^{2}+a-b^{2}}{(1+a) a}\right)^{h^{\prime}} & \text { for } h^{\prime}<0\end{cases}
$$

if $a^{2}+a-b^{2}>0$ and $a^{2}+a+b+2 a b \leqslant 0$.

By symmetry of the kernel $K(\cdot, \cdot)$, it can easily be calculated in the domain $h^{\prime}<h$.

We write

$$
A \stackrel{\mathrm{df}}{=} \frac{(a+b)(1+a+b)}{a^{2}+a+b+2 a b} \quad \text { and } \quad B \stackrel{\mathrm{df}}{=} \frac{a^{2}+a-b^{2}}{(1+a) a} .
$$

The Euler equality will be solved for each kernel separately.

For the kernel (3.2), the equality is of the form

$$
\begin{aligned}
& {\left[\int_{-\vartheta}^{0} \mu\left(h^{\prime}\right) d h^{\prime}+\int_{0}^{h} A^{h^{\prime}} \mu\left(h^{\prime}\right) d h^{\prime}+A^{h} \int_{h}^{T-\vartheta} \mu\left(h^{\prime}\right) d h^{\prime}\right] \mathbf{1}(h)+} \\
& +\left[B^{h} \int_{-\vartheta}^{h} \mu\left(h^{\prime}\right) d h^{\prime}+\int_{h}^{0} B^{h^{\prime}} \mu\left(h^{\prime}\right) d h^{\prime}+\int_{0}^{T-\vartheta} \mu\left(h^{\prime}\right) d h^{\prime}\right] \mathbf{1}(-h)=h
\end{aligned}
$$


where 1(h) is the function of unit jump. Equality (3.6) will be solved separately for $h \leqslant 0$ and $h \geqslant 0$, and then we shall "join" both solutions at the point $l_{b}=0$. The only condition for joining them is the equality of the solutions at this point.

For $h \leqslant 0$, by (3.6) we have

$$
B^{h} \int_{-\infty}^{h} \mu\left(h^{\prime}\right) d h^{\prime}+\int_{h}^{0} B^{h^{\prime}} \mu\left(h^{\prime}\right) d h^{\prime}=h-C_{1}
$$

for $h \in[-\vartheta, 0]$, where

$$
C_{1} \stackrel{d t}{=} \int_{0^{+}}^{T-\theta} \mu\left(h^{\prime}\right) d h^{\prime}
$$

is unknown. Differentiating (3.7) twice with respect to $h$ we get one of the possible solutions: $\mu(h)=-B^{-h}$ for $h \in(-\vartheta, 0)$. The function will be extended for the points $h=-\vartheta$ and $h=0$ by means of point measures so that equality (3.7) will hold for $h=0$ and $h=-\vartheta$. We obtain

$$
\mu(h)=-B^{-h}+C_{-\vartheta} \delta(h+\vartheta)+C_{01} \delta(h), \quad h_{b} \in[-\vartheta, 0],
$$

where $\delta(\cdot)$ is the Dirac function and the constants are equal to

$$
C_{-\vartheta}=B^{\vartheta} / \ln B \text { and } C_{01}=-C_{1}-1 / \ln B .
$$

Putting (3.8) into (3.7) we check that the function (3.8) is the solution of the equality.

For $h \geqslant 0$, equality (3.6) is of the form

$$
\int_{0}^{h} A^{h^{\prime}} \mu\left(h^{\prime}\right) d h^{\prime}+A^{h} \int_{h}^{T-\vartheta} \mu\left(h^{\prime}\right) d h^{\prime}=h-C_{2}, \quad h \in[0, T-\vartheta],
$$

where

$$
C_{2} \stackrel{d t}{=} \int_{-0}^{0-} \mu(h) d h .
$$

Comparing (3.8) and (3.10) we obtain

$$
C_{2}=1 / \ln B \text {. }
$$

Equality (3.9) can be solved in the same way as (3.7). Now, we get

$$
\mu(h)=A^{-h}+C_{T-\vartheta} \delta(h-T+\vartheta)+C_{02} \delta(h), \quad h_{b} \in[0, T-\vartheta]
$$

where

$$
C_{T-\theta}=\frac{1}{\ln A} A^{-(T-\theta)} \quad \text { and } \quad C_{02}=-C_{2}-\frac{1}{\ln A} .
$$


Putting (3.11) into the last equality we obtain

$$
C_{02}=\frac{-1}{\ln B}-\frac{1}{\ln A}
$$

Having the function $\mu(\cdot)$ defined on the interval $(0, T-\vartheta]$, we calculate $C_{1}$ and $C_{01}$ :

$$
C_{1}=1 / \ln A \quad \text { and } \quad C_{01}=-1 / \ln A-1 / \ln B .
$$

It can be noticed that $C_{01}=C_{02}=-1 / \ln A-1 / \ln B \stackrel{d f}{=} C_{0}$.

The equality of the constants $C_{01}$ and $C_{02}$ is the sufficient condition to join the solutions (3.8) and (3.12) at the point $h=0$. Therefore, the obtained solution of equality (3.6) is of the form

$$
\begin{aligned}
\mu(h)=-B^{-h} \cdot \mathbf{1}(-h)+ & C_{-\vartheta} \delta(h+\vartheta)+ \\
& +C_{0} \delta(h)+C_{T-\vartheta} \delta(h-T+\vartheta)+A^{-h} \cdot \mathbf{1}(h)
\end{aligned}
$$

for $h \in[-\vartheta, T-\vartheta]$, where

$$
C_{-\vartheta}=\frac{B^{\vartheta}}{\ln B}, \quad C_{0}=\frac{-1}{\ln A}-\frac{1}{\ln B}, \quad C_{T-\vartheta}=\frac{1}{\ln A} A^{-(T-\vartheta)} .
$$

The Euler equality will now be solved for the kernel (3.3). The value of the functional $\Phi_{2}$ (from (2.4)) equals $+\infty$ for each function $\mu(\cdot)$ which fulfils the restrictions and which does not equal zero in the interval $[-\vartheta, 0]$. Then

$$
\frac{\left(\Phi_{1}[\mu]\right)^{2}}{\Phi_{2}[\mu]}=0
$$

The supremum (2.2) is calculated with all the functions $\mu(\cdot)$ fulfilling (2.3) and $\left(2.3^{\prime}\right)$ and such that $\mu(h)=0$ for $h \in[-\vartheta, 0)$. It is then the problem identical with the problem $(2.2),(2.3),\left(2.3^{\prime}\right)$ in which the subset $[0, T-\vartheta]$ is taken now from the set $\Theta_{\vartheta}$.

From (2.6) we obtain the Euler equality in the form

$$
\int_{0}^{h} A^{h^{\prime}} \mu\left(h^{\prime}\right) d h^{\prime}+A^{h} \int_{h}^{T-\vartheta} \mu\left(h^{\prime}\right) d h^{\prime}=h, \quad h \in[0, T-\vartheta] .
$$

We obtain the solution of this equality using the solution of equality (3.9) for $C_{2}=0$ :

$$
\mu(h)=A^{-h}+C_{0}^{\prime} \delta(h)+C_{T-\vartheta} \delta(h-T+\vartheta), \quad h \in[0, T-\vartheta],
$$

where $C_{0}^{\prime}=-1 / \ln A$ and $C_{T-\vartheta}=A^{-(T-v)} / \ln A$. 
The Euler equality is solved in the same way for the kernel (3.4). The solution takes the form

$$
\mu(h)=-B^{-h}+C_{-\vartheta} \delta(h+\vartheta)+C_{0}^{\prime \prime} \delta(h), \quad h \in[-\vartheta, 0],
$$

where $C_{0}^{\prime \prime}=-1 / \ln B$ and $C_{-\vartheta}=B^{\vartheta} / \ln B$.

According to [2], the solution of (3.6) appears to be not only a necessary condition but also a sufficient one for finding the supremum (2.2). Moreover, the solutions (3.13), (3.14), and (3.15) satisfy (2.3) and (2.3'). In this way they are the solutions of the problem of finding the supremum (2.2) with conditions (2.3) and (2.3'). The solutions can be represented as the result of subtraction of two densities, which follows from the property of (2.2), formulated after formula (2.6). Therefore, these solutions give such a supremum in formula (2.2). We shall calculate the value of this supremum.

Introducing the solution of Euler's equality into the functional $\Phi_{2}$, by (2.4) and (2.6) we obtain

$$
\Phi_{2}[\mu]=\int_{\Theta_{\vartheta}} \int_{\Theta_{\vartheta}} K\left(h, h^{\prime}\right) \mu(h) \mu\left(h^{\prime}\right) d h d h^{\prime}=\int_{\Theta_{\vartheta}} h \mu(h) d h
$$

The extreme value of functional (2.2) is therefore equal to

$$
\sup _{\mu^{\prime}} \frac{\left(\Phi_{1}\left[\mu^{\prime}\right]\right)^{2}}{\Phi_{2}\left[\mu^{\prime}\right]}=\int_{\Theta_{\vartheta}} h \mu(h) d h .
$$

Introducing (3.13), (3.14), and (3.15) into (3.16) we get the accurate lower variance bound of the disorder moment estimator for the process under consideration. Depending on the values of the intensity coefficients $a$ and $b$ in (1.1), this limit equals

$$
\frac{1}{\ln ^{2} A}\left(1-A^{-(T-\vartheta)}\right)+\frac{1}{\ln ^{2} B}\left(1-B^{\vartheta}\right)
$$

where $a^{2}+a-b^{2}>0, a^{2}+a+b+2 a b>0 ;$

$$
\begin{gathered}
\frac{1}{\ln ^{2} A}\left(1-A^{-(T-\vartheta)}\right), \quad \text { where } a^{2}+a-b^{2} \leqslant 0 ; \\
\frac{1}{\ln ^{2} B}\left(1-B^{\vartheta}\right), \quad \text { where } a^{2}+a-b^{2}>0, a^{2}+a+b+2 a b \leqslant 0 ;
\end{gathered}
$$

$A$ and $B$ being defined by (3.5). 
Obviously, this limit depends on the process parameters $(a, b, \vartheta)$ and on the time of observation $T$. When the factors $\vartheta$ and $T-\vartheta$ increase, the minimal variance increases to the limit

$$
\begin{gathered}
1 / \ln ^{2} A+1 / \ln ^{2} B, \quad \text { where } a^{2}+a-b^{2}>0, a^{2}+a+b+2 a b>0 ; \\
1 / \ln ^{2} A, \quad \text { where } a^{2}+a-b^{2} \leqslant 0 \\
1 / \ln ^{2} B, \quad \text { where } a^{2}+a-b^{2}>0, a^{2}+a+b+2 a b \leqslant 0 .
\end{gathered}
$$

4. Finally, we consider the situation in which we have the possibility to observe independent channels, each of which is described by a negative binomial process similar to the one presented above. We assume that the intensity is changed stepwise simultaneously in all channels at a moment $\vartheta \in[0, T]$,

$$
\gamma_{s}(t / \vartheta)=a_{s}+b_{s} \cdot 1(t-\vartheta), \quad a_{s}>0, b_{s}>0
$$

where $s$ is a number of channels $(s=1, \ldots, n)$.

According to the considerations in Section 2, we may state that if $X$ is a random vector with independent components, the kernel (2.5) can be calculated by the formula.

$$
\begin{aligned}
K\left(h, h^{\prime}\right) & =\int_{\mathscr{X}}(n) \int_{\mathscr{X}} \frac{f\left(x_{1}, \ldots, x_{n} ; \vartheta+h\right) f\left(x_{1}, \ldots, x_{n} ; \vartheta+h^{\prime}\right)}{f\left(x_{1}, \ldots, x_{n} ; \vartheta\right)} d x_{1} \ldots d x_{n} \\
& =\prod_{i=1}^{n} \int_{\mathscr{X}} \frac{f_{i}\left(x_{i} ; \vartheta+h\right) f_{i}\left(x_{i} ; \vartheta+h^{\prime}\right)}{f_{i}\left(x_{i} ; \vartheta\right)} d x_{i}=\prod_{i=1}^{n} K_{i}\left(h, h^{\prime}\right)
\end{aligned}
$$

Where $K_{i}(\cdot, \cdot)$ is the kernel related to the $i$-th component of the vector $X$, having density $f_{i}$, and $f$ denotes density of the vector $X$. Since the channels are independent, the density function in this case is the product of densities (1.3) that describe single channels. Therefore, the value of the kernel at each point equals the product of kernel values at that point, calculated for a single channel. Making calculations similar to those for the one-dimensional case, we can evaluate the minimal estimator variance of the parameter $\vartheta$ which uses information about all $n$ channels.

Defining new constants by the formulae

$$
A=\prod_{s=1}^{n} \frac{\left(a_{s}+b_{s}\right)\left(1+a_{s}+b_{s}\right)}{a_{s}^{2}+a_{s}+b_{s}+2 a_{s} b_{s}} \quad \text { and } \quad B=\prod_{s=1}^{n} \frac{a_{s}^{2}+a_{s}-b_{s}^{2}}{\left(1+a_{s}\right) a_{s}}
$$


we can see that the minimal variance is equal to

$$
\frac{1}{\ln ^{2} A}\left(1-A^{-(T-\vartheta)}\right)+\frac{1}{\ln ^{2} B}\left(1-B^{\vartheta}\right)
$$

where $a_{s}^{2}+a_{s}-b_{s}^{2}>0$ for all $s \in\{1, \ldots, n\}$, and

$$
\frac{1}{\ln ^{2} A}\left(1-A^{-(T-\vartheta)}\right)
$$

where $a_{s}^{2}+a_{s}-b_{s}^{2} \leqslant 0$ for some $s \in\{1, \ldots, n\}$.

It can be easily checked that in the described multi-dimensional case the minimal estimator variance of the parameter $\vartheta$ is smaller than the minimal estimator variance that uses information from a single channel.

5. The precise lower variance bound of the disorder moment estimator for the Poisson process has been obtained in [2]. The multi-dimensional case can be analyzed for this process similarly as in Section 4 . We observe channels each of which is described by a Poisson process. We assume that the intensity is changed stepwise simultaneously in all channels at the moment $\vartheta \in[0, T]$ :

$$
\lambda_{s}(t / \vartheta)=a_{s}+b_{s} \cdot 1(t-\vartheta)
$$

where $s$ is a number of channels $(s=1, \ldots, n)$.

Pertel' [2] has obtained the lower variance bound for the one-dimensional case in the form

$$
\left(\frac{a^{2}}{b^{2}}\right)^{2}\left[1-\exp \left(-\frac{b^{2}}{a} \vartheta\right)\right]+\left(\frac{a+b}{b^{2}}\right)^{2}\left[1-\exp \left(-\frac{b^{2}}{a+b}(T-\vartheta)\right)\right]
$$

Calculating similarly as in Section 4, we obtain the following lower variance bound of the estimator for the multi-dimensional case:

$$
\begin{aligned}
& \frac{1}{\left(\sum_{s=1}^{n} b_{s}^{2} / a_{s}\right)^{2}}\left[1-\exp \left(-\sum_{s=1}^{n} \frac{b_{s}^{2}}{a_{s}} \vartheta\right)\right]+ \\
& +\frac{1}{\left(\sum_{s=1}^{n} b_{s}^{2} /\left(a_{s}+b_{s}\right)\right)^{2}}\left[1-\exp \left(-\sum_{s=1}^{n} \frac{b_{s}^{2}}{a_{s}+b_{s}}(T-\vartheta)\right)\right]
\end{aligned}
$$




\section{References}

[1] J. Kiefer, On minimum variance estimators, Ann. Math. Statist. 23 (1952), p. 627-629.

[2] V. A. Pertel' (В. A. П е р т е ль), Точнал ниянял еранича дисперсии оченки момента равладки пуассоновского потока, Техническая кщбернетика 5 (1971), p. $167-170$.

INSTITUTE OF MATHEMATICS

TECHNICAL UNIVERSITY OF WROCEAW

50-370 WROCEAW

Received on 16. 10. 1981;

revised version on 10. 5. 1982 